\title{
Características clínicas de los pacientes con apneas obstructivas del sueño: diferencias según género
}

\author{
CONSTANZA SALAS C. $* * * * * *$, JORGE DREYSE D. $* * * * * *$, M. FRANCISCA OLIVARES C.*******, \\ ANDREA CONTRERAS S.**,*****, GONZALO NAZAR M.***,*****, GLORIA RIBALTA L.***,*****, \\ GONZALO LABARCA T. $* * * *, * * * * *$, M. FRANCISCA LETELIER D.*,***** y JORGE JORQUERA A.*,*****
}

Clinical features in patients with obstructive sleep apnea syndrome: differences by gender

Introduction: Obstructive sleep apnea (OSA) is more prevalent in men, population studies show a ratio of 2:1, however in clinical studies the frequency is as high as 6:1. These differences in the clinical setting may be a consequence of variations in the reporting of symptoms in men compared to women. Objective: To evaluate gender differences between women and men with recent diagnosis of OSA. Patients: A cross-sectional, study of patients undergoing home sleep apnea testing (HSAT) with clinical suspicion of OSA. Demographic, anthropometric, comorbidities and HSAT variables were collected. We performed t student analysis, Mann-Whitney test or chi square test as appropriate. Results: 1,044 patients were included: mean age $53.2 \pm 14$ years, $76 \%$ men. Women with OSA have a higher BMI (32.2 \pm 6.1 vs $30.8 \pm 5.0, p=0.002)$, were older $(61.4 \pm 12.2$ vs 52.6 $\pm 13.9, p<0.001)$, but have a lower neck circumference $(N C)(38.1 \pm 3.6$ vs. $43.2 \pm 3.3, p<0.001)$. The women presented lower AHI and shorter duration of apneas. Although the classic symptoms of apnea and daytime sleepiness showed no differences, women reported more frequently insomnia, morning headache, depression and use of hypnotic drugs. Conclusions: Clinical differences between gender are present at time of diagnosis. Woman are older and more obese, although they have a lower NC. They have a milder disease, but they refer to be more tired, headache, insomnia and depression. We must be alert in the different clinical presentation of women to improve the diagnostic suspicion.

Key words: Sleep apnea, obstructive; humans; males; females; antropometry; sleep; comorbidity.

\section{Resumen}

Introducción: El síndrome de apnea obstructiva del sueño (SAHOS) es más prevalente en hombres, los estudios poblacionales muestran una relación de 2:1, sin embargo, en los estudios clínicos la frecuencia llega a ser de hasta de 6:1. Estas diferencias en el ambiente clínico puede ser consecuencia de variaciones en el reporte de síntomas en hombres comparados con mujeres. Objetivo: Evaluar las diferencias de género en la presentación clínica de apnea obstructiva de sueño. Pacientes: Estudio transversal de pacientes sometidos a una poligrafia respiratoria (PR) con sospecha clínica de $S A H O S$. Se recolectaron datos demográficos, antropométricos, comorbilidades y las variables de la PR. Se realizó estudio t de student, Mann-Whitney y chi-cuadrado según correspondiera. Resultados: Se incluyeron 1.044 pacientes: edad promedio 53,2 \pm 14 años, $76 \%$ hombres. Las mujeres con SAHOS poseen mayor $\operatorname{IMC}(32,2 \pm 6,1$ vs 30,8 $\pm 5,0 ; p=0,002)$ y edad $(61,4 \pm 12,2$ vs 52,6 $\pm 13,9 ; p<0,001)$, pero menor circunferencia de cuello (CC) (38,1 $\pm 3,6$ vs 43,2 $\pm 3,3 ; p<0,001)$, además, presentan menor IA/H y menor duración de las apneas. No hubo diferencias en los síntomas clásicos de apneas presenciada y somnolencia diurna, sin embargo, describen más frecuentemente insomnio, cefalea matinal, depresión y uso de fármacos hipnóticos. Conclusiones: Las mujeres al momento del diagnóstico son de mayor edad y más obesas, aunque presentan una CC menor, presentan una enfermedad más leve, pero refieren

Centro de Enfermedades Respiratorias,

** Neurología.

*** Otorrinolaringología.

**** Complejo Asistencial Víctor Ríos Ruiz, Los Ángeles.

***** Grupo de Estudio Trastornos Respiratorios del Sueño (GETRS), Clínica Las Condes. 
más fatiga, cansancio, cefalea e insomnio. Debemos estar alerta en la presentación clínica diferente de las mujeres para mejorar la sospecha diagnóstica.

Palabras clave: Apnea obstructiva del sueño; seres humanos; hombres; mujeres; antropometría; sueño; comorbilidad.

\section{Introducción}

El síndrome de apnea e hipopnea obstructiva del sueño (SAHOS) produce como síntoma cardinal somnolencia diurna excesiva ${ }^{1}$, y se ha asociado a mayor riesgo de hipertensión arterial $^{2-5}$, accidentes de tránsito ${ }^{6-7}$ y mayor número de eventos cardiovasculares fatales y no fata$\operatorname{les}^{8-10}$. Una revisión sistemática reciente de los estudios epidemiológicos de apnea del sueño ${ }^{11}$ mostró una prevalencia de SAHOS (Índice de apnea $\geq 5$ eventos/hora) de $22 \%$ (rango 9 a $37 \%$ ) en hombres y $17 \%$ (4 a 50\%) en mujeres, con una relación entre hombres y mujeres de 2:1. Existe además una asociación entre la menopausia y el desarrollo del SAHOS, donde la prevalencia aumenta significativamente a cifras similares a los hombres ${ }^{14-15}$. Esta distinta prevalencia se debe a diferencias genéticas, hormonales, anatómicas, a nivel de la estructura craneofacial y de la vía aérea superior, y a la distinta distribución de la grasa $^{18-19}$. Sin embargo, en estudios clínicos de pacientes que consultan en las clínicas de sueño muestran una relación de hasta $6: 1^{16-17}$. Esta diferencia puede estar reflejada en variaciones respecto al reporte de síntomas, presentación clínica, diferente tolerancia a los síntomas o desconocimiento de una presentación clínica distinta por parte de los equipos de salud. El objetivo del presente trabajo es evaluar las diferencias de género en la presentación clínica de los pacientes con síndrome de apnea obstructiva de sueño.

\section{Pacientes y Métodos}

Estudio transversal, retrospectivo, donde se evaluaron pacientes adultos sometidos a estudio de sueño por la sospecha clínica de SAHOS (presencia de síntomas de ronquido, apneas observadas por terceros o somnolencia diurna excesiva) que fueron derivados para realizar un estudio de sueño mediante poligrafía respiratoria (PR) ambulatoria. Previo a la PR se aplicó encuesta estandarizada de síntomas, con el propósito de evaluar el horario de sueño, grado de somnolencia diurna, ronquido, apneas presenciadas, insomnio, episodios de sofocación nocturna, nicturia, cefalea matinal, boca seca al despertar, pérdida de memoria y deterioro cognitivo. Se consignaron los datos sociodemográficos, hábitos, comorbi- lidades y los datos antropométricos, tales como peso, talla y circunferencia cervical, abdominal y de cintura, entre otras. Se recolectaron datos demográficos, antropométricos, comorbilidades y las diferentes variables de la PR (índice de apnea hipopnea $(\mathrm{IA} / \mathrm{H})$, saturación media y mínima de oxígeno, tiempo con saturación bajo $90 \%$, tiempo total de registro). A todos los pacientes se les aplicó las encuestas del estudio, que incluía la escala de somnolencia de Epworth ${ }^{20}$, escala de Thornton modificado, STOP-BANG ${ }^{13}$ y se calculó el puntaje del modelo predictivo de Flemons (circunferencia cervical ajustada $)^{21}$. El estudio fue aprobado por el Comité de Ética de la institución y los pacientes firmaron el acta de consentimiento informado previo a su incorporación.

\section{Poligrafía respiratoria}

Para la realización de la poligrafía respiratoria (PR) se utilizó un equipo marca Embletta, Embletta Gold y Embletta MPR (Embla Systems, Natus sleep products, EE.UU) que cumple con las exigencias de la Academia Americana de Medicina del Sueño (AASM) para los estudios de nivel III $^{22}$, que incluía el registro de flujo nasal mediante cánula nasal conectada a un transductor de presión, movimientos tóraco-abdominales (xtrace RIP belts: pletismografía de inductancia), frecuencia cardiaca y saturación de oxígeno a través de un oxímetro de pulso digital y sensor de posición. Los estudios fueron realizados en el domicilio, previa instrucción en el laboratorio para la instalación a cargo del paciente o de su familia. Se les entregó un instructivo impreso con información iconográfica del procedimiento de instalación. Todos los registros se descargaron al día siguiente. El registro debía cumplir con los estándares exigidos por la AASM para estudios diagnósticos, en caso contrario era repetido. El análisis de la PR fue realizado manualmente por un único médico especialista en enfermedades respiratorias con amplia experiencia en el diagnóstico de trastornos respiratorios del sueño, de acuerdo a las normas de la $\mathrm{AASM}^{22}$. Se emplearon las siguientes definiciones: Se definió apnea como la ausencia de flujo por más de 10 segundos: a) Apnea obstructiva: ausencia del flujo oronasal en presencia de movimientos tóraco-abdominales; b) Apnea central: ausencia 
de flujo oronasal y de los movimientos tóracoabdominales; c) Apnea mixta: ausencia de flujo oronasal que comienza con un componente central y termina como obstructiva; y d) Hipopneas: reducción del flujo aéreo discernible (más de 30\% y menos del $90 \%$ ) por un período superior a 10 segundos. Para el análisis manual, si había pérdida de la señal de flujo, usado primariamente para detectar las apneas e hipopneas, se utilizaba el canal alternativo del esfuerzo tóraco-abdominal. Todos los eventos respiratorios debían acompañarse de una caída de la saturación de oxígeno de al menos 3\%. Se calculó el número de eventos respiratorios dividiendo el número total de eventos respiratorios por el tiempo total de registro en horas (número de apneas más número de hipopneas dividido por tiempo total de registro). Los hallazgos fueron considerados diagnósticos de SAHOS cuando el número de eventos respiratorios fue mayor o igual a cinco eventos por hora.

\section{Análisis estadístico}

Los resultados fueron expresados como valores promedio \pm desviación estándar para las variables medidas en escala numérica, como mediana más rango intercuartil 25-75 para las variables ordinales y en número y porcentaje para las medidas en escala nominal. Las variables cualitativas fueron comparadas mediante la prueba de Chi cuadrado, las ordinales con test $U$ de Mann-Whitney, y las variables continuas con la prueba t de Student. Como gold estándar se utilizó el resultado de la poligrafía respiratoria (PR), siendo aquellos con PR normal la población de referencia. Los análisis y el registro de los datos se realizaron mediante el software Excel 2016 y el software SPSS 15.0 (SPSS Inc., Chicago). Se consideró significativo estadísticamente un valor de $\mathrm{p} \leq 0,05$.

\section{Resultados}

Se evaluaron mediante poligrafía respiratoria 1.044 sujetos adultos con sospecha clínica de SAHOS: El 85,8\% de los sujetos estudiados (896/1044) presentaron diagnóstico de SAHOS. La edad promedio fue 53,2 \pm 14 años, $24 \% \mathrm{mu}$ jeres, el índice de masa corporal (IMC) de 30,6 $\pm 5,4 \mathrm{~kg} / \mathrm{m}^{2}$ y la circunferencia cervical de 42,1 $\pm 3,9 \mathrm{~cm}$. De los pacientes con SAHOS, 29,2\% eran leves, $29,3 \%$ moderados y $41,6 \%$ graves. En la Tabla 1 se describen las características clínicas y de la poligrafía respiratoria de los pacientes normales y con SAHOS incluidos en el estudio.
Los síntomas clásicos de apnea observada por testigos y somnolencia diurna excesiva no presentaron diferencias entre hombres y mujeres; sin embargo, las mujeres describen más frecuentemente insomnio (39\% versus 24\%; $p<$ $0,001)$, presencia de ronquido $(23 \%$ versus $17 \%$, $\mathrm{p}=0,017)$, cefalea matinal $(51 \%$ versus $34 \%$, $\mathrm{p}<0,001)$, historia de depresión $(41 \%$ versus $11 \%, \mathrm{p}<0,001)$ y cansancio al despertar. Las mujeres además presentaban mayor prevalencia de hipotiroidismo (36\% versus $9 \%, \mathrm{p}<0,001)$, diabetes mellitus $(26 \%$ versus $17 \%, \mathrm{p}=0,003)$, uso de hipnóticos $(14 \%$ versus $9 \%, \mathrm{p}=0,011)$ y de antidepresivos $(29 \%$ versus $12 \%, \mathrm{p}=0,009)$. Las mujeres con SAHOS eran de mayor edad $(61,4 \pm 12,2$ versus $52,6 \pm 13,9$ años; $\mathrm{p}<0,001)$ e $\operatorname{IMC}\left(32,2 \pm 6,1\right.$ versus $30,8 \pm 5,0 \mathrm{~kg} / \mathrm{m}^{2} ; \mathrm{p}=$ $0,002)$, pero menor circunferencia de cuello $(38,1$ $\pm 3,6$ vs 43,2 $\pm 3,3 \mathrm{~cm} ; \mathrm{p}<0,001)$. En la Tabla 2 se describen las características antropométricas, clínicas, comorbilidades y resultados de los cuestionarios de sueño en hombres y mujeres. Las mujeres presentan enfermedad menos grave (IA/H: 27,5 $\pm 19,6$ versus $31,1 \pm 21,9$ eventos/ hora; $\mathrm{p}=0,042)$ y menor duración de las apneas $(18,9 \pm 6,2$ versus $20,3 \pm 4,7 \mathrm{~s} ; \mathrm{p}=0,001)$, pero con mayor caída de la saturación de oxígeno. La Tabla 3 muestra las principales características de la poligrafía respiratoria en hombres y mujeres.

\section{Discusión}

Los principales hallazgos del presente estudio son: a) Las mujeres al momento del diagnóstico son de mayor edad y más obesas, aunque presentan una circunferencia cervical menor; b) Las mujeres presentan una enfermedad más leve, pero refieren más fatiga y cansancio y presentan más insomnio y depresión.

Las mujeres al momento del diagnóstico presentan mayor edad que los hombres con una diferencia de casi 9 años $(61,4$ versus 52,6 años), a pesar que existe discrepancia con otros estudios $^{23-26}$, las mujeres chilenas de esta serie se diagnostican en promedio una década después de la edad de la menopausia. Además, el momento del diagnóstico es casi 10 años después que otros estudios, Alotair y cols ${ }^{27}$ estudiaron a 384 sujetos en forma consecutiva, la edad promedio de diagnóstico fue a los 53,9 años. La explicación del por qué las mujeres chilenas consultan en forma más tardía, podría ser en parte debido a que presentan una presentación clínica diferente, desconocimiento del equipo médico de esta presentación clínica distinta o tener menor acceso a los métodos diagnósticos. La obesidad, factor 
Tabla 1. Características clínicas y de la poligrafía respiratoria de los sujetos normales y con SAHOS

\begin{tabular}{|c|c|c|c|}
\hline & $\begin{array}{c}\text { Normales } \\
(n=148)\end{array}$ & $\begin{array}{l}\text { SAHOS } \\
(n=895)\end{array}$ & valor de $p$ \\
\hline \multicolumn{4}{|c|}{ Demográficos y clínicos } \\
\hline Edad, años & $45,4 \pm 16,4$ & $54,5 \pm 14,0$ & $<0,001$ \\
\hline Circunferencia cervical, $\mathrm{cm}$ & $38,3 \pm 4,1$ & $42,1 \pm 3,9$ & $<0,001$ \\
\hline Índice de masa corporal, $\mathrm{kg} / \mathrm{m}^{2}$, & $27,0 \pm 4,7$ & $31,1 \pm 5,3$ & 0,002 \\
\hline $\mathrm{PAS}, \mathrm{mmHg}$ & $118 \pm 14$ & $125 \pm 17$ & $<0,001$ \\
\hline $\mathrm{PAD}, \mathrm{mmHg}$ & $76 \pm 10$ & $80 \pm 11$ & $<0,001$ \\
\hline $\mathrm{SpO}_{2}, \%$ & $96,1 \pm 1,8$ & $95,3 \pm 2,6$ & 0,001 \\
\hline Índice paquete año (ex y fumadores) & $9,4 \pm 12,3$ & $20,1 \pm 24,6$ & 0,002 \\
\hline \multicolumn{4}{|c|}{ Cuestionarios de sueño } \\
\hline Epworth & $8(5-13)$ & $9(5-13)$ & 0,211 \\
\hline STOP-Bang & $4(3-5)$ & $6(5-6)$ & $<0,001$ \\
\hline Flemons & $44(39-47)$ & $49(46-52)$ & $<0,001$ \\
\hline Thornton & $10(7-16)$ & $14(9-19)$ & $<0,001$ \\
\hline \multicolumn{4}{|c|}{ Variables de la poligrafía respiratoria } \\
\hline Tiempo total registro, $\mathrm{min}$ & $466 \pm 84$ & $463 \pm 76$ & 0,723 \\
\hline $\mathrm{IA} / \mathrm{H}, \mathrm{ev} / \mathrm{h}$ & $2,9 \pm 2,1$ & $30,3 \pm 21,4$ & $<0,001$ \\
\hline Duración media apneas, $\mathrm{s}$ & $17,1 \pm 3,9$ & $20,0 \pm 5,1$ & $<0,001$ \\
\hline ID- $4, \mathrm{ev} / \mathrm{h}$ & $3,9 \pm 6,6$ & $27,7 \pm 21,4$ & $<0,001$ \\
\hline $\mathrm{SpO}_{2}$ promedio, $\%$ & $94,4 \pm 1,8$ & $92,1 \pm 3,3$ & $<0,001$ \\
\hline $\mathrm{SpO}_{2}$ mínima, $\%$ & $87,5 \pm 8,2$ & $78,0 \pm 9,6$ & $<0,001$ \\
\hline СТ-90\%, \% & $2,4 \pm 11,0$ & $16,7 \pm 23,9$ & $<0,001$ \\
\hline \multicolumn{4}{|l|}{ Gravedad } \\
\hline SAHOS Leve & & $261(29,2)$ & \\
\hline SAHOS Moderado & & $262(29,3)$ & \\
\hline SAHOS Grave & & $372(41,5)$ & \\
\hline
\end{tabular}

Variables se expresan como: categóricas n (\%); ordinales mediana (cuartiles 25 - 75); continuas promedio \pm desviación estándar. PAS: Presión arterial sistólica; PAD: Presión arterial diastólica; IA/H: Î́ndice de apneas/hipopneas; ID4: Índice de desaturación $\geq 4 \%, \mathrm{SpO}_{2}$ : Saturación de pulso de oxígeno; CT 90\%: Tiempo saturación de oxígeno bajo 90\%, SAHOS: Síndrome de apneas/hipopneas obstructivas del sueño.

predisponente de apnea del sueño, en nuestro estudio las mujeres tenían un índice de masa corporal significativamente mayor que los hombres. Muchos estudios han mostrado que las mujeres son más obesas que los hombres ${ }^{24,28-30}$, e incluso la cohorte de Wisconsin mostró que las mujeres tienen un IMC mayor al mismo nivel de índice de eventos respiratorios ${ }^{31}$. Los síntomas clásicos de apneas presenciadas y somnolencia diurna excesiva no presentaron diferencias entre mujeres y hombre similar a otros estudios ${ }^{24,32}$, sin embargo, las mujeres describen más frecuentemente insomnio, cefalea matinal, cansancio al despertar y presentan más historia de depresión con el consiguiente mayor uso de drogas hipnóticas y antidepresivas. Similar a lo comunicado por Young y cols., las mujeres más frecuentemente reportan depresión, ansiedad y cefalea matinal ${ }^{24,33}$. Esto podría explicar la dificultad o retraso en el diagnóstico de las mujeres con SAHOS al atribuirse parte de los síntomas a trastornos de ansiedad o depresión. La frecuencia de insomnio en mujeres es el doble que en el hombre (40\% vs 24\%; p < $0,001)$, similar a otras series donde las mujeres describen mayores problemas en conciliar el sueño ${ }^{27}$ y además describen mayor cansancio al despertar, tal como Ambrogetti y cols. ${ }^{34}$ y Pillar y Lavie ${ }^{35}$, quienes reportan más fatiga e insomnio comparado con los hombres. Estos hallazgos son importantes ya que en las mujeres con insomnio debemos evaluar la presencia o no de apnea del sueño. El mayor reporte de ronquido habitualmente se asocia a los hombres debido a las diferencias de la distribución de la grasa a nivel de la vía aérea superior, sin embargo, en esta serie estaba más presente en las mujeres. Las mujeres presentan más frecuentemente hipotiroidismo (34\% vs 9\%) y diabetes mellitus (26\% versus $17 \%, \mathrm{p}=0,003)$ lo cual es concordante con reportes previos que encuentra que las mujeres con hipotiroidismo tienen mayor riesgo relativo de presentar SAHOS. No encontramos diferencias al momento del diagnóstico con otras comorbilidades cardiovasculares. Nuestros resultados 
Tabla 2. Características clínicas, antropométricas y de cuestionarios de sueño de los sujetos con SAHOS según sexo.

\begin{tabular}{|c|c|c|c|}
\hline & $\begin{array}{l}\text { Hombres } \\
(n=703)\end{array}$ & $\begin{array}{l}\text { Mujeres } \\
(n=192)\end{array}$ & valor de $p$ \\
\hline \multicolumn{4}{|c|}{ Características demográficas y clínicas } \\
\hline Edad, años & $52,6 \pm 13,9$ & $61,4 \pm 12,2$ & $<0,001$ \\
\hline Circunferencia cervical, cm & $43,2 \pm 3,3$ & $38,1 \pm 3,6$ & $<0,001$ \\
\hline Índice de masa corporal, $\mathrm{kg} / \mathrm{m}^{2}$ & $30,8 \pm 5,0$ & $32,2 \pm 6,1$ & 0,002 \\
\hline $\mathrm{PAS}, \mathrm{mmHg}$ & $125 \pm 16$ & $125 \pm 19$ & 0,976 \\
\hline $\mathrm{PAD}, \mathrm{mmHg}$ & $80 \pm 12$ & $79 \pm 11$ & 0,382 \\
\hline $\mathrm{SpO}_{2}, \%$ & $95,5 \pm 2,4$ & $94,8 \pm 3,1$ & 0,004 \\
\hline Tabaquismo (ex y actual) & $368(52)$ & $82(43)$ & 0,013 \\
\hline Índice paquete año (ex y fumadores) & $19 \pm 24$ & $24 \pm 23$ & 0,101 \\
\hline Hipertensión arterial & $298(42)$ & $119(62)$ & $<0,001$ \\
\hline Reflujo gastroesofágico & $326(46)$ & $102(53)$ & 0,047 \\
\hline Diabetes mellitus tipo 2 & $120(17)$ & $50(26)$ & 0,003 \\
\hline Cardiopatía coronaria & $78(11)$ & $14(7)$ & 0,080 \\
\hline Accidente cerebrovascular & $33(5)$ & $8(4)$ & 0,465 \\
\hline Asma & $61(9)$ & $22(11)$ & 0,140 \\
\hline Enfermedad Pulmonar Obstructiva Crónica & $31(4)$ & $14(7)$ & 0,079 \\
\hline Depresión & $79(11)$ & $79(41)$ & $<0,001$ \\
\hline Uso antidepresivos & $84(12)$ & $56(29)$ & $<0,001$ \\
\hline Insomnio & $166(24)$ & $75(39)$ & $<0,001$ \\
\hline Uso hipnóticos & $61(9)$ & $27(14)$ & 0,011 \\
\hline Uso relajantes musculares & $11(2)$ & $7(4)$ & 0,056 \\
\hline Hipotiroidismo & $61(9)$ & $69(36)$ & $<0,001$ \\
\hline Dislipidemia & $195(28)$ & $59(31)$ & 0,105 \\
\hline Ronquido habitual & $123(17)$ & $44(23)$ & 0,017 \\
\hline Apneas observadas (a diario) & $330(47)$ & $71(37)$ & 0,080 \\
\hline Cansancio al despertar & $453(64)$ & $136(72)$ & 0,038 \\
\hline Cefalea matinal & $237(34)$ & $97(51)$ & $<0,001$ \\
\hline \multicolumn{4}{|c|}{ Cuestionarios de sueño } \\
\hline Epworth & $9(5-13)$ & $9(6-14)$ & 0,181 \\
\hline STOP-Bang & $6(5-6)$ & $5(4-5)$ & $<0,001$ \\
\hline Flemons & $50(47-53)$ & $45(42-48)$ & $<0,001$ \\
\hline Thornton & $13(9-18)$ & $15(8-20)$ & 0,089 \\
\hline
\end{tabular}

Variables se expresan como: categóricas n (\%); ordinales mediana (cuartiles 25 - 75); continuas promedio \pm desviación estándar; PAS: Presión arterial sistólica; PAD: Presión arterial diastólica; $\mathrm{SpO}_{2}$ : Saturación de pulso de oxígeno.

concuerdan con otros reportes donde describen que las mujeres presentan menor índice de apneas que los hombres ${ }^{23,29,36,38}$, con una menor duración de los eventos respiratorios. Es posible que las mujeres sean más sintomáticas a menor gravedad de la condición ${ }^{31}$ y que podría ser explicado por un aumento de los eventos respiratorios principalmente durante el sueño REM ${ }^{38}$.

La debilidad de este trabajo es que los datos fueron obtenidos durante la entrevista inicial y que puede ser afectada por el nivel educacional del paciente, la presencia de miembros de la familia y principalmente del esposo(a) que podría haber afectado la respuesta de algunas preguntas. El entrevistador obviamente no era ciego y esto puede constituir un sesgo. La población estudiada puede representar un potencial error en la evaluación de la fuerza de los distintos cuestionarios para identificar a los sujetos con riesgo de apnea del sueño. Sin embargo, los pacientes a quienes se les solicitaba la PR eran derivados de las especialidades que habitualmente evalúan o sospechan este trastorno ( $53 \%$ especialista en enfermedades respiratorias, $23 \%$ otorrinolaringólogos, 9\% cardiólogos y un porcentaje menor médicos internistas, diabetólogos y neurólogos entre otros) y por lo tanto representa bastante bien la práctica médica habitual. Dentro de las fortalezas de este estudio están: a) El tamaño muestral; b) Fue realizado en una población de pacientes adultos ambulatorios con sospecha de apneas del sueño en un ambiente clínico real. 
Tabla 3. Características de la poligrafía respiratoria de los sujetos con SAHOS según sexo

\begin{tabular}{|c|c|c|c|}
\hline & $\begin{array}{c}\text { Hombres } \\
(n=703)\end{array}$ & $\begin{array}{l}\text { Mujeres } \\
(n=192)\end{array}$ & valor de $p$ \\
\hline \multicolumn{4}{|c|}{ Características demográficas y clínicas } \\
\hline Tiempo total registro, $\min$ & $462 \pm 77,7$ & $468 \pm 73,2$ & 0,350 \\
\hline $\mathrm{IA} / \mathrm{H}, \mathrm{ev} / \mathrm{h}$ & $31,1 \pm 21,9$ & $27,5 \pm 19,6$ & 0,042 \\
\hline Duración media apneas, seg & $20,3 \pm 4,7$ & $18,9 \pm 6,2$ & 0,001 \\
\hline ID-4, ev/h & $27,6 \pm 20,9$ & $28,2 \pm 22,4$ & 0,760 \\
\hline $\mathrm{SpO}_{2}$ promedio, $\%$ & $92,2 \pm 3,0$ & $91,8 \pm 4,1$ & 0,117 \\
\hline $\mathrm{SpO}_{2}$ mínima, $\%$ & $78,3 \pm 9,3$ & $76,9 \pm 10,6$ & 0,062 \\
\hline СТ-90\%, \% & $15,5 \pm 22,2$ & $21,2 \pm 28,9$ & 0,004 \\
\hline Diagnóstico & & & 0,091 \\
\hline SAHOS Leve & $191(27,2)$ & $70(36,5)$ & \\
\hline SAHOS Moderado & $215(30,6)$ & $47(24,5)$ & \\
\hline SAHOS Grave & $297(42,2)$ & $75(39,0)$ & \\
\hline
\end{tabular}

Las variables continuas se expresan como promedio \pm desviación estándar y las variables categóricas como número y porcentaje; IA/H: Índice de apneas/hipopneas; ID-4: Índice de desaturación $\geq 4 \%, \mathrm{SpO}_{2}$ : Saturación de pulso de oxígeno; CT 90\%: Tiempo saturación de oxígeno bajo 90\%, SAHOS: Síndrome de apneas/hipopneas obstructivas del sueño.

En resumen, el presente estudio muestra que las mujeres al momento del diagnóstico son de mayor edad y más obesas, aunque presentan una circunferencia cervical menor; presentan una enfermedad de menor gravedad, pero refieren más fatiga, cefalea, cansancio y presentan más insomnio y depresión. Este es el primer trabajo que describe la influencia del sexo en la presentación clínica del síndrome de apnea obstructiva del sueño en la población chilena y debemos estar alerta ante estas diferencias con el fin de aumentar la sospecha diagnóstica.

\section{Bibliografía}

1.- BALDWIN CM, GRIFFITH KA, NIETO FJ, O'CONNOR GT, WALSLEBEN JA, REDLINE S.

The association of sleep-disordered breathing and sleep symptoms with quality of life in the Sleep Heart Health Study. Sleep 2001; 24: 96-105.

2.- PEPPARD PE, YOUNG T, PALTA M, SKATRUD J. Prospective study of the association between sleepdisordered breathing and hypertension. New Eng J Med 2000; 342: 1378-84.

3.- DURÁN-CANTOLLA J, AIZPURU F, MARTÍNEZNULL C, BARBÉ ILLA F. Obstructive sleep apnea/ hypopnea and systemic hypertension. Sleep Med Rev 2009; 13: 323-31.

4.- CANO-PUMAREGA I, DURÁN-CANTOLLA J, AIZPURU F, MIRANDA-SERRANO E, RUBIO R, MARTÍNEZ-NULL C, et al. On behalf of the Spanish Sleep and Breathing Group. Obstructive sleep apnea and systemic hypertension: Longitudinal study in the general population: The Vitoria Sleep Cohort. Am J
Respir Crit Care Med 2011; 184: 1299-304.

5.- MARIN JM, AGUSTI A, VILLAR I, FORNER M, NIETO D, CARRIZO SJ, BARBÉ, et al. Association between treated and untreated obstructive sleep apnea and risk of hypertension. JAMA 2012; 307: 2169-76.

6.- YOUNG T, BLUSTEIN J, FINN L, PALTA M. Sleepdisordered breathing and motor vehicle accidents in a population-based sample of employed adults. Sleep 1997; 20: 608-13.

7.- TERÁN-SANTOS J, JIMÉNEZ-GÓMEZ A, CORDERO-GUEVARA J. The association between sleep apnea and the risk of traffic accidents. Cooperative Group Burgos-Santander. N Engl J Med 1999; 340: 847-51.

8.- MARSHALL NS, WONG KK, LIU PY, CULLEN SR, KNUIMAN MW, GRUNSTEIN RR. Sleep apnea as an independent risk factor for all-cause mortality: The Busselton Health Study. Sleep 2008; 31: 1079-85.

9.- MARIN JM, CARRIZO SJ, VICENTE E, AGUSTÍ AG. Long-term cardiovascular outcomes in men with obstructive sleep apnoea-hypopnoea with or without treatment with continuous positive airway pressure: an observational study. Lancet 2005; 365: 1046-53.

10.- YOUNG T, FINN L, PEPPARD PE, SZKLO-COXE M, AUSTIN D, NIETO FJ, et al. Sleep-disorderedbreathing and mortality: eigtheen-year follow-up of the Wisconsin Sleep Cohort. Sleep 2008; 31: 1071-8.

11.- FRANKLIN KA, LINDBERG E. Obstructive sleep apnea is a common disorder in the population-a review on the epidemiology of sleep apnea. J Thorac Dis 2015; 7(8): 1311-22.

12.- CARRILlO J, VARGAS C, CISTERNAS A, TIRADO P. Prevalencia de Riesgo de Apnea Obstructiva del Sueño en Población Adulta Chilena. Rev Chil Enferm Respir 2016, 32: S 57 (CL*70). 
13.- CHUNG F, YEGNESWARAN B, LIAO P, CHUNG SA, VAIRAVANATHAN S, ISLAM S, et al. STOP questionnaire: a tool to screen patients for obstructive sleep apnea. Anesthesiology 2008; 108: 812-21.

14.- YOUNG T, FINN L, AUSTIN D, PETERSON A. Menopausal status and sleep-disordered breathing in the Wisconsin Sleep Cohort Study. Am J Respir Crit Care Med 2003; 167: 1181-5.

15.- TISHLER PV, LARKIN EK, SCHLUCHTER MD, REDLINE S. Incidence of sleep-disordered breathing in an urban adult population: the relative importance of risk factors in the development of sleep-disordered breathing. JAMA 2003; 289: 2230-2237.

16.- EVANS J, SKOMRO R, DRIVER H, GRAHAM B, MAYERS I, MCRAE L, et al. Sleep laboratory test referrals in Canada: sleep apnea rapid response survey. Can Respir J 2014; 21: e 4-10.

17.- LARSSON LG, LINDBERG A, FRANKLIN KA, LUNDBACK B. Gender differences in symptoms related to sleep apnea in a general population and in relation to referral to sleep clinic. Chest 2003; 124: 204-11.

18.- SEE CQ, MENSAH E, OLOPADE CO. Obesity, ethnicity, and sleep-disordered breathing: medical and health policy implications. Clin Chest Med 2006; 27: 521-533.

19.- VILLANUEVA AT, BUCHANAN PR, YEE BJ, GRUNSTEIN RR. Ethnicity and obstructive sleep apnoea. Sleep Med Rev 2005; 9: 419-36.

20.- JOHNS MW. A new method for measuring daytime sleepi ness: the Epworth sleepiness scale. Sleep 1991; 14(6): 540-5.

21.- FLEMMONS WW. Obstructive Sleep apnea. N Engl J Med 2002; 347: 498-501.

22.- RAMACHANDRAN SK, JOSEPHS LA. A metaanalysis of clinical screening tests for obstructive sleep apnea. Anesthesiology 2009; 110: 928-39.

23.- MOHSENIN V. Gender differences in the expression of sleep-disordered breathing: role of upper airway dimensions. Chest 2001; 120: 1442-7.

24.- YOUNG T, HUTTON R, FINN L, BADR S, PALTA M. The gender bias in sleep apnea diagnosis. Are women missed because they have different symptoms? Arch Intern Med 1996; 156: 2445-51.

25.- MORRELL MJ, FINN L, MCMILLAN A, PEPPARD PE. The impact of ageing and sex on the association between sleepiness and sleep disordered breathing. Eur Respir J 2012; 40: 386-93.

26.- CAIRNS A, POULOS G, BOGAN R. Sex differences in sleep apnea predictors and outcomes from home sleep apnea testing. Nat Sci Sleep 2016; 8: 197-205.

27.- ALOTAIR H, BAHAMMAM A. Gender differences in Saudi patients with obstructive sleep apnea. Sleep Breath 2008; 12: 323-9.

28.- YOUNG T, PALTA M, DEMPSEY J, SKATRUD J, WEBER S, BADR S. The occurrence of sleep-disordered breathing among middle-aged adults. N Engl J Med 1993; 328: 1230-5.

29.- LEECH JA, ONAL E, DULBERG C, LOPATA MA. A comparison of men and women with occlusive sleep apnea syndrome. Chest 1988; 94: 983-8.

30.- SMITH R, RONALD J, DELAIVE K, WALLD R, MANFREDA J, KRYGER MH. What are obstructive sleep apnea patients being treated for prior to this diagnosis? Chest 2002; 121: 164-72.

31.- YOUNG T, EVANS L, FINN L, PALTA M. Estimation of the clinically diagnosed proportion of sleep apnea syndrome in middle-aged men and women. Sleep 1997; 20: 705-6

32.- SHEPERTYCKY MR, BANNO K, KRYGER MH Differences between men and women in the clinical presentation of patients diagnosed with obstructive sleep apnea syndrome. Sleep 2005; 28: 309-14.

33.- GREENBERG-DOTAN S, REUVENI H, SIMONTUVAL T, OKSENBERG A, TARASIUK A. Gender differences in morbidity and health care utilization among adult obstructive sleep apnea patients. Sleep 2007; 30: 1173-80

34.- AMBROGETTI A, OLSON LG, SAUNDERS NA. Differences in the symptoms of men and women with obstructive sleep apnoea. Aust NZ J Med 1991; 21: 863 6.

35. PILLAR G, LAVIE P. Psychiatric symptoms in sleep apnea syndrome: effects of gender and respiratory disturbance index. Chest 1998; 114: 697-703.

36. O'CONNOR C, THORNLEY KS, HANLY PJ. Gender differences in the polysomnographic features of obstructive sleep apnea. Am J Respir Crit Care Med 2000; 161: 1465-72.

37.- MOLINE ML, BROCH L, ZAK R, GROSS V. Sleep in women across the life cycle from adulthood through menopause. Sleep Med Rev 2003; 7: 155-77.

38.- WARE JC, MCBRAYER RH, SCOTT JA. Influence of sex and age on duration and frequency of sleep apnea events. Sleep 2000; 23: 165-70.

Correspondencia a:

Dr. Jorge Jorquera Arévalo

Centro de Enfermedades Respiratorias.

Grupo de estudio trastornos respiratorios del sueño

(GETRS).

Clínica Las Condes.

Lo Fontecilla 441 Las Condes

Santiago, Chile.

Email: jjorquera@clc.cl 